\title{
An ultrastructural study of spermatogenesis and sperm morula breakdown in Arenicola marina (L.) (Annelida: Polychaeta)
}

\author{
A. A. Pacey* \& M. G. Bentley** \\ Gatty Marine Laboratory, Department of Biology and Pre-clinical Medicine, University of \\ St. Andrews; St. Andrews, Fife, KY16 8LB, Scotland, U.K.
}

\begin{abstract}
Spermatogenesis in the lugworm Arenicola marina, in common with other members of Arenicolidae, occurs in the coelomic fluid and results in the formation of discs of mature spermatozoa known as a morulae. Within a morula, individual spermatozoa are connected by a common mass of cytoplasm called the cytophore and therefore make up a syncitium. Immediately prior to spawning, and in response to an endocrine substance known as "Sperm Maturation Factor" (SMF), the structure of the sperm morulae breaks down and free spermatozoa are liberated. These are subsequently spawned from the body cavity. The investigation described here uses transmission electron microscopy to investigate the ultrastructural changes, which accompany spermatogenesis and the breakdown of sperm morulae in response to SMF in vitro. The study demonstrates that the cytophore appears to have a key role both during spermatogenesis and during sperm morula breakdown. The ultrastructure of sperm morulae and of mature spermatozoa is described. The structure of spermatozod is shown to be primitive with a single flagellum which appears to be coiled at its distal end. The phagocytosis of free spermatozoa by coelomocytes is also described and it is suggested that these may play a role in the resorption of unspawned gametes in vivo.
\end{abstract}

\section{INTRODUCTION}

The reproductive biology of Arenicola marina (the Lugworm) is well documented and has been described in a number of papers such as those of Newell (1948), Duncan (1960), and Howie $(1959,1984)$. Often, it is characterised by having a distinct spawning crisis and, at most localities in the U.K. which have been investigated, this usually occurs in the late autumn or early winter. Gametogenesis occurs in the preceding months and whilst the proliferation of spermatogonia occurs in the testes, spermatogonia are released at an early stage to develop in the body cavity bathed in coelomic fluid (Ashworth, 1904; Olive, $1972 \mathrm{a}, \mathrm{b})$. The spermatozoa of $A$. marina, along with other members of the Arenicolidae, have a classical primitive structure (see Franzén, 1956 for sperm types), and spermatogenesis within this family is particularly interesting because it results in the formation of a disc of mature spermatozoa called a morula (see Newell, 1948; Olive, 1983; Sawada, 1984). Within a morula, individual spermatozoa are cytoplasmically connected

\footnotetext{
- Present address: Station Zoologique, URA 671 CNRS, B.P. 28, F-06230 Villefranche-sur-Mer, France

* Addressee for all correspondence
} 
to a central mass of cytoplasm called the cytophore. The precise function of the cytophore remains unknown, although it has been described in a number of polychaete families including the Terebellidae (Eckelbarger, 1975; Smith, 1989) and in the Opheliidae (Ochi et al., 1977).

When present in sperm morulae, the spermatozoa of $A$. marina are incapable of being spawned to the exterior of the body cavity (Howie, 1961). Prior to spawning it is therefore necessary for the morulae structure to dissociate and for spermatozoa to become free. The process of sperm morula breakdown is under endocrine control from a factor released from the prostomium (Howie, 1963) which has been termed "Sperm Maturation Factor" (SMF) (Bentley, 1985). Various aspects of this process have been investigated (see Howie, 1961, 1963; Bentley, 1985; Bentley et al., 1990) and others are currently the focus of attention (Bentley \& Pacey, 1991). This study, however, investigates the ultrastructure of mature spermatozoa in $A$. marina using transmission electron microscopy and observes the ultrastructural changes which occur both during spermatogenesis, and during the liberation of free sperm from sperm morulae.

Earlier studies have investigated some aspects of sperm ultrastructure in A. marina. Meijer (1979), for example, published micrographs of sperm morula in $A$. marina but said very little of the ultrastructural detail of the morula or of mature spermatozoa. The dissociation of sperm morulae has been described previously using light microscopy (Bentley, 1986a), and preliminary ultrastructural observations using transmission electron microscopy have been made (Bentley, 1986b). However, a detailed study of both spermatogenesis and sperm morula breakdown using scanning electron microscopy has been carried out (Bentley \& Pacey, 1989) and this is a complementary study to that work.

\section{MATERIALS AND METHODS}

Fixations were carried out on sperm samples taken from animals which had been collected from a number of sites around the U.K. Details of collection sites, maintenance of animals in the laboratory and removal of sperm samples from the coelom have been described previously (Bentley \& Pacey, 1989).

The fixation method was also as described previously, except that samples were dehydrated through an acetone series before being placed in an araldite:acetone mixture $(4: 1 \mathrm{v} / \mathrm{v})$ overnight and then embedded in araldite. This was allowed to polymerise at $60{ }^{\circ} \mathrm{C}$ for 36 hours. Sections were cut on a A. F. Huxley pattern ultramicrotome and mounted on G300 mesh copper grids (EM Scope). Prior to observation, the sections were double stained according to the methods of Lewis \& Knight (1977); for 10-15 minutes with saturated uranyl acetate in $70 \%$ ethanol and then, following washing, with Reynolds' lead citrate (Reynolds, 1963) for 10-15 minutes. Sections were examined using a Phillips EM301 transmission electron microscope,

Sperm samples for transmission electron microscopy were incubated with prostomial SMF in vitro and sequential fixations were carried out at five minute intervals. During each experiment, thirteen activation and thirteen control incubations were carried out in parallel and sperm were incubated with either $500 \mu \mathrm{l}$ of prostomial homogenate at a final concentration of 0.4 prostomia $\cdot \mathrm{ml}^{-1}$, or $500 \mu \mathrm{l} \mathrm{TFSW}$ (Triple filtered seawater) respectively, at a temperature of $14^{\circ} \mathrm{C}$. All incubations were carried out in Eppendorf tubes.

At time zero, $50 \mu \mathrm{l}$ of "dry sperm" (undiluted coelomic sperm) was added to each of 
the incubations and at five minute intervals for 60 minutes, one experimental and one control incubation were fixed by the addition of fixative and processed for electron microscopy as described above. Five experiments were carried out which gave a total of 130 fixed sperm samples. In addition, coelomic samples were taken from male A. marina which were not yet sexually mature (therefore containing early stages of spermatogenesis), and these were fixed for transmission electron microscopy in the same way.

\section{RESULTS}

\section{Spermatogenesis}

Following the release of spermatogonia from the testis in Arenicola marina, one of the earliest stages observed in fixed samples of the coelomic fluid is shown in Figure 1. This shows a transverse section through a spherical cluster of spermatogonia with individual gonia connected by cytoplasmic bridges. The exact size of the cluster and presumably the number of gonia which it contains is variable, since this depends upon the total number of divisions which have taken place prior to fixation. The cluster shown in Figure 1 is ca $30 \mu \mathrm{m}$ in diameter. It is often difficult to ascertain the exact stage of spermatogenesis of such clusters; whether they are spermatocytes (i.e. are still undergoing proliferative mitotic division) or whether they are spermatids (i.e. have undergone their final meiotic division). The transverse section shown in Figure 2, however, is clearly that of a cluster of developing spermatids. Within this cluster, the nucleus of individual spermatids are elongate and are undergoing nuclear condensation. In some spermatids, vesicles are clearly visible and in one, a centriole is present. The cytoplasmic connections between the spermatids have become thickened in comparison with the earlier stage shown in Figure 1.

As differentiation of the spermatids continues, the cluster flattens and becomes disclike. Spermatids acquire a longitudinal axis and a polarity with the rudiments of a flagellum becoming visible at their distal end. Figure 3 shows a transverse section through a disc at this stage of development. Within these spermatids nuclear condensation is partially complete and the development of sperm organelles is occurring. Rudimentary mitochondria are forming $(\mathrm{m})$ and pre-acrosomal vesicles $(\mathrm{p})$ are often visible. The cytophore appears to play a key role in the development of the spermatids, and in later stages it is seen to contain many active cell organelles. Figure 4 shows spermatids which have completed nuclear condensation and in which the acrosomal vesicle, although not yet fully differentiated, has migrated to the anterior end of the spermatozoon. At this stage, endoplasmic reticulum (er) is widely distributed throughout the cytoplasm of the cytophore and suggests that active synthesis is occurring within the sperm disc. Electron dense spherical vesicles are also visible within the cytophore, although it is not clear whether these are lysosomes or storage organelles.

As the development into mature spermatozoa proceeds, the cytophore shrinks in size until it contains very few cytoplasmic inclusions apart from membrane bound vesicles. Figure 5 is of a mature sperm morula and it is within this structure that the spermatozoa remain until just prior to spawning or when incubated in vitro with SMF. 


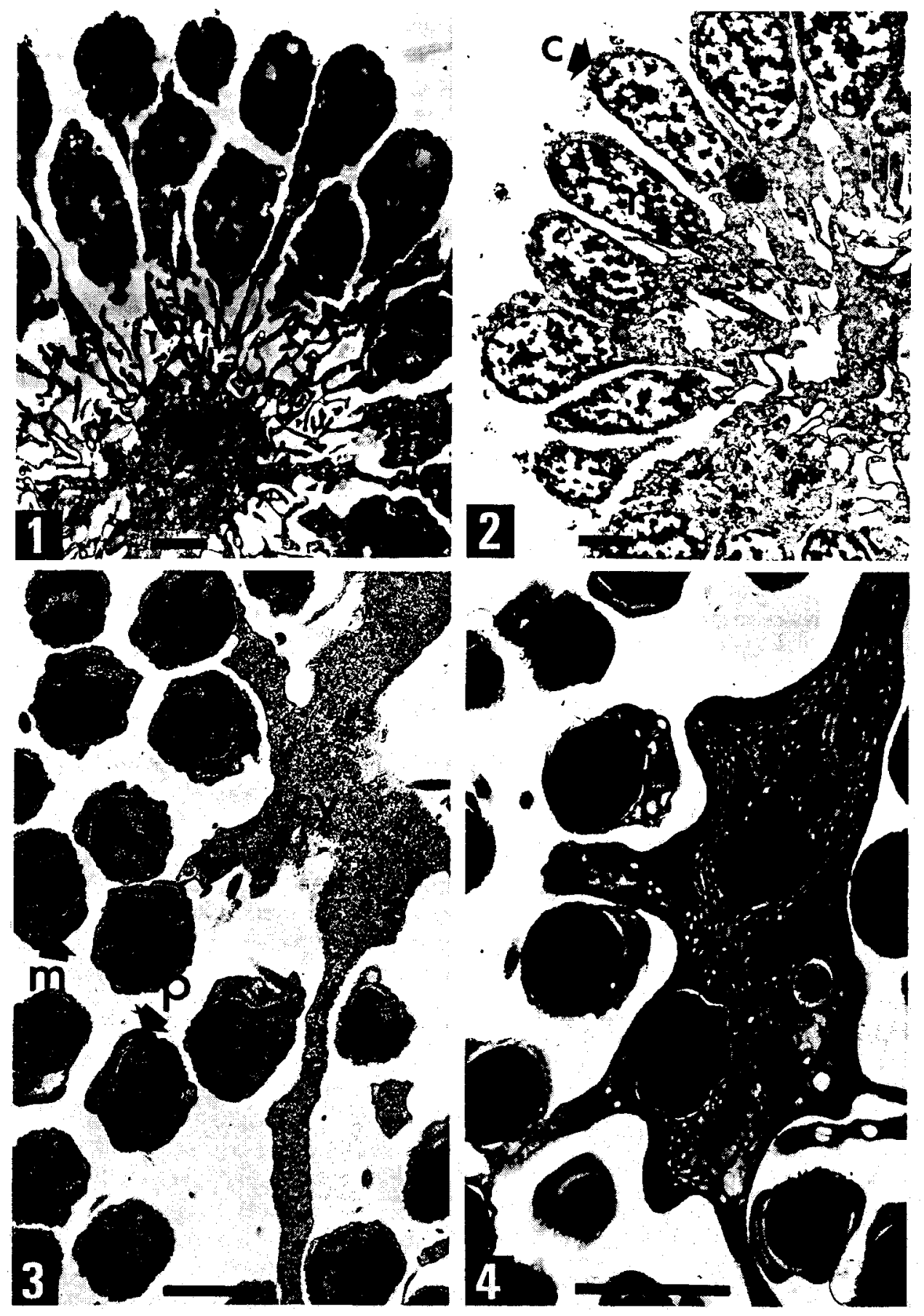




\section{Ultrastructure of mature spermatozoa and the sperm morula}

Figure 5 shows a transverse section through a fully differentiated sperm morula and the orientation of mature spermatozoa with their acrosomes toward the cytophore. It can be seen that the cytophore contains very little cytoplasm and few cytoplasmic inclusions, but it is seen to make connection with individual sperm heads. The details of these connections are seen more clearly in Figure 6, with a stalk of cytoplasm from the cytophore making contact with individual spermatozoa on the lateral surface of the sperm head, just behind the acrosome. Figure 6 also shows detail of the head and midregion of a mature spermatozoon.

The spermatozoa of Arenicola marina conform to a typical primitive ultrastructure. The head region of mature spermatozoa consists mainly of nuclear material surmounted by a cup-like acrosome (Fig. 6) (see Sawada [1984] for acrosome types). At the apex of the acrosome is a small acrosomal button or apical vesicle. This is anterior to the acrosomal vesicle which lies in the space between the acrosome and the nucleus, and in $A$. marina contains material which is moderately granular. The nucleus, both in transverse and longitudinal section, appears ovoid in shape and posteriorly there are four to six mitochondria. Figure 7 shows a transverse section through the mid-region of a spermatozoon in which there are five. Located within the mid-region of spermatozoa are two centrioles oriented at $90^{\circ}$ to each other (Figs 8, 9). The distal centriole lies along the central vertical axis of the spermatozoon and has nine paddle-like arms. From these arise the nine outer doublets of the flagellar axoneme, the two central singlets arising distal to the centriolar apparatus (Fig. 10).

The sperm flagellum of $A$. marina is relatively unmodified along its length and consists of the conventional arrangement of $9+2$ microtubules tightly enclosed within a membrane sheath. Occasionally, however, modifications of the flagellum are observed. These usually occur with regard to the membrane surrounding the axoneme which may become flared, expanded, or occasionally forms are observed where two axonemes are surrounded by a single membrane. Transverse sections through various points along the distal region of the flagellum suggest that the axoneme is coiling within the flagellar part of the cell membrane (Fig. 11).

\section{Incubation of sperm morulae with SMF in vitro}

When coelomic sperm samples of Arenicola marina are incubated with SMF in vitro, free sperm are liberated from the sperm morulae after approximately 50 minutes at $14^{\circ} \mathrm{C}$

Fig. 1. Transverse section through a cluster of developing spermatocytes or spermatids showing cytoplasmic connections between individual cells which connect in the centre of the cluster to make up the cytophore (cy). Scale bar $=10 \mu \mathrm{m}$

Fig. 2. Transverse section of a cluster of developing spermatids. Nuclear condensation is partially complete and organelles such as centrioles are visible $(\mathrm{n}=$ nucleus; $\mathrm{c}=$ centriole). Scale bar $=2 \mu \mathrm{m}$ Fig. 3. Transverse section through a later stage of morula development, the cluster of spermatids now having flattened to form a disc. The differentiation of spermatozoa is now occurring and cell organelles are clearly visible $(\mathrm{m}=$ mitochondria $\mathrm{p}=$ pre-acrosomal vesicle; $\mathrm{cy}=$ cytophore). Scale bar $=2 \mu \mathrm{m}$

Fig. 4. Spermatid disc in which nuclear condensation is complete. The cytophore contains endoplasmic reticulum (er) which suggests active biosynthesis is occurring. Scale bar $=2 \mu \mathrm{m}$ 



Fig. 5. Structure of mature sperm morula showing the orientation of mature spermatozoa to the cytophore. The sperm remain bound together in this way until just prior to spawning or following incubation with SMF in vitro. Scale bar $=10 \mu \mathrm{m}$

Fig. 6. Ultrastructure of acrosome and attachment of mature spermatozoa in the cytophore $a=$ acrosome; av = acrosomal vesicle; att = cytoplasmic attachment of spermatozoa to the cytophore). Scale bar $=1 \mu \mathrm{m}$

Fig. 7. Mid-region of mature spermatozoa showing position of distal centriole. Scale bar $=0.2 \mathrm{um}$ Fig. 8. Longitudinal section of mature spermatozoa showing proximal centriole (arrowed). Scale bar $=0.2 \mu \mathrm{m}$

Fig. 9. Mid-region of mature spermatozoa showing distal centriole (arrowed). Scale bar $=0.2 \mu \mathrm{m}$ Fig. 10. Mid-region of mature spermatozoon showing position of centriolar apparatus and the outer doublet (od) and central singlet (cs) microtubules of the flagellar axoneme. Scale bar $=0.2 \mu \mathrm{m}$ 


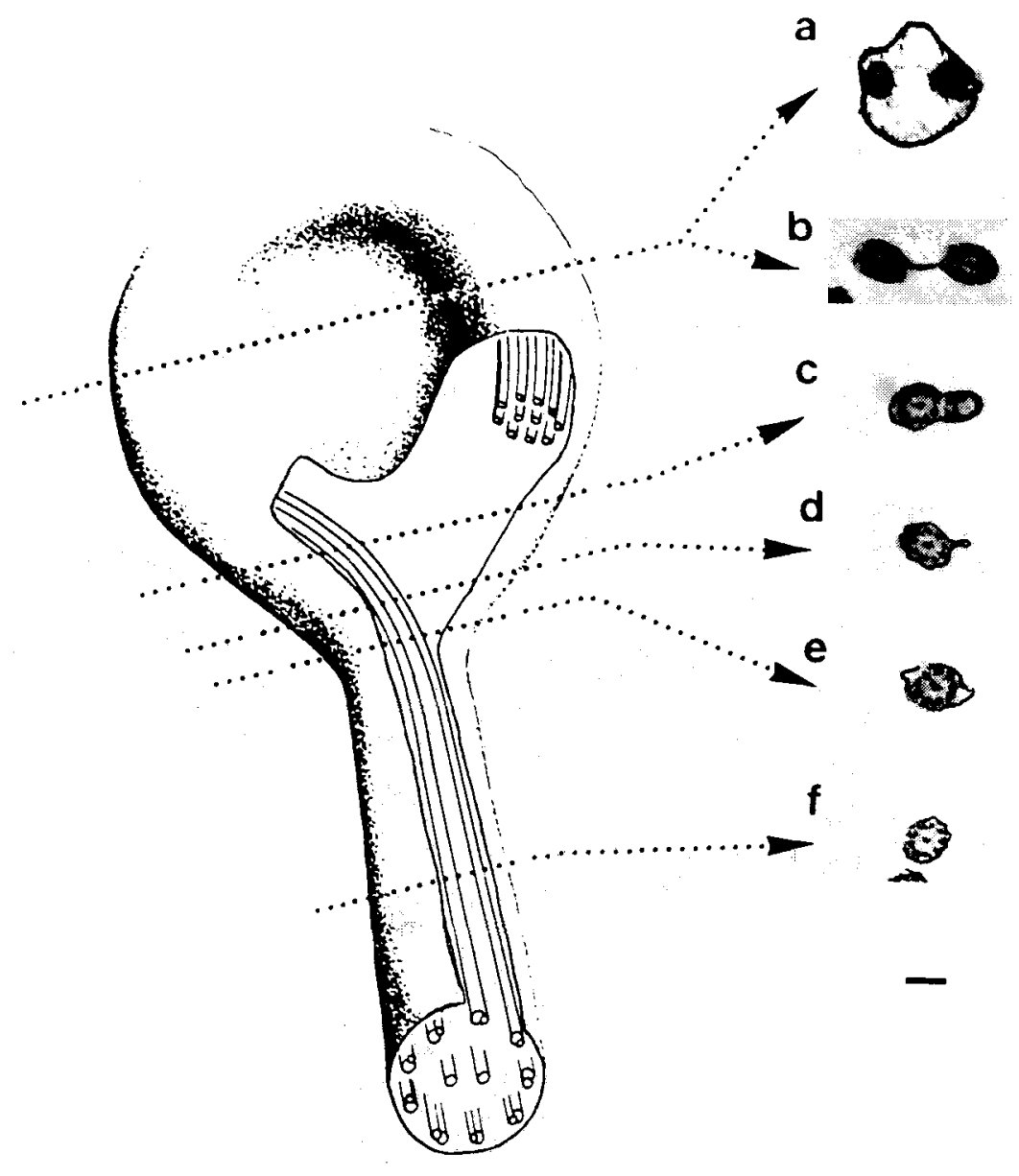

11

Fig. 11. Diagrammatic representation of the distal end of mature sperm flagellum showing the coiling of the axoneme within an expanded portion of the flagellar membrane. The diagram shows the membrane tightly adhering to the microtubules (Section b), although Section a shows an alternate form, where the membrane is expanded. The other transverse sections (c-f) correspond to interpretation by the diagram. Scale bar $=0.2 \mu \mathrm{m}$

(Bentley, 1985). Sperm morulae present within sperm samples which are fixed at the commencement of incubation, show no ultrastructural changes and have an appearance unchanged from that of the unactivated morulae. Only after twenty minutes of incubation with prostomial extracts do any changes become apparent (Fig. 12). Figure 12 shows that significant changes have taken place with respect to the state of the cytophore. At this time the cytophore becomes swollen and significantly increases in size (cf. Fig. 6). The stalks of cytoplasm which connect the cytophore to the spermatozoa and the spermatozoa 


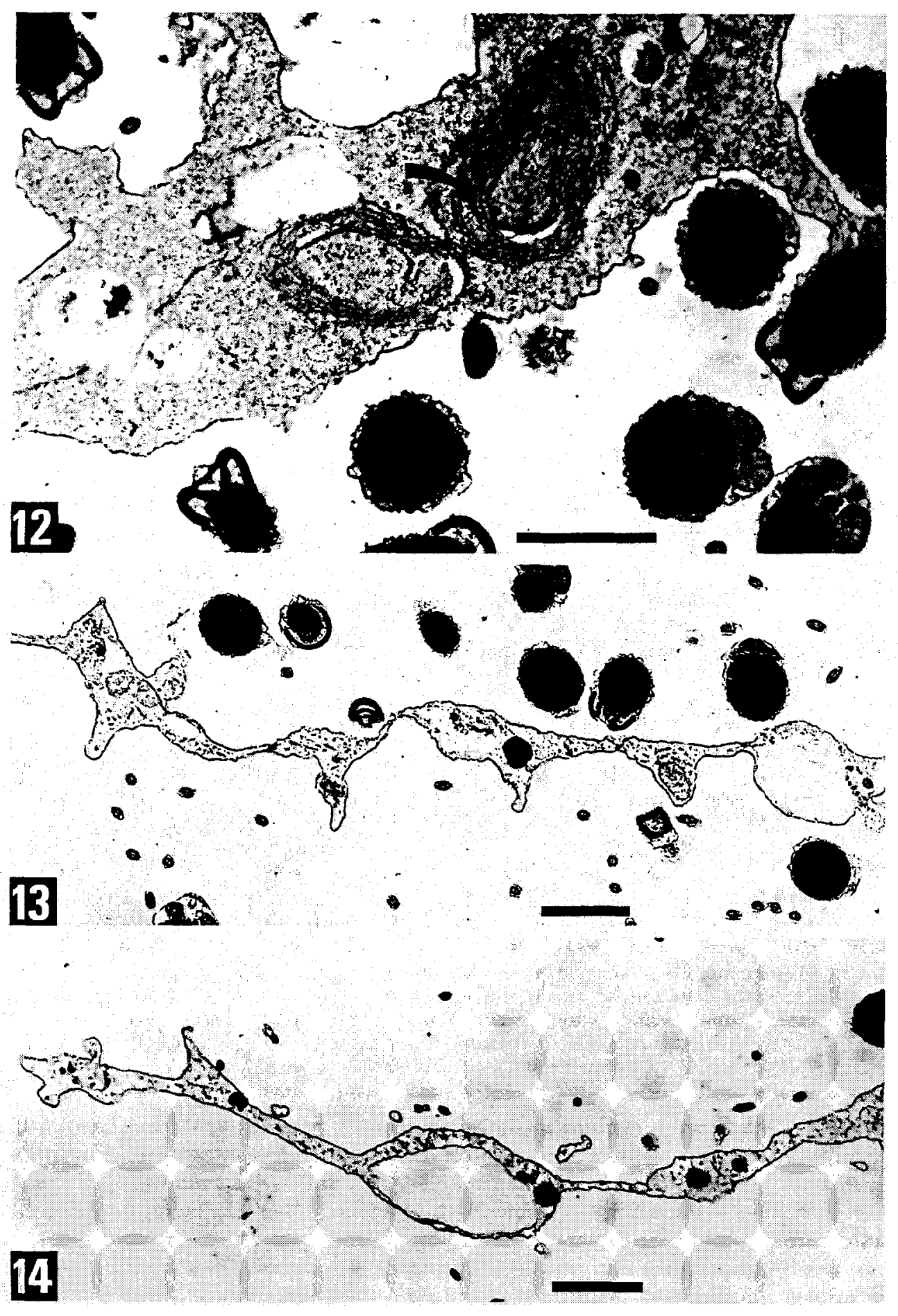


to each other, are similarly swollen. Both the cytophore and the connecting stalks are filled with a granular electron dense material, and whorls of membrane within the cytoplasm are visible (arrowed).

After 30 minutes incubation, fewer sperm heads are observed being associated with the cytophore (Fig. 13). The cytophore at this time is very swollen, contains some clear vacuoles, and appears to be undergoing degeneration. After 40 minutes observation, many remnant cytophores are visible (Fig. 14). Observations of live sperm incubations at this time shows that although individual flagella are beating, few sperm are free and a degree of structural integrity between spermatozoa which make up a morula is still maintained. The observations described here indicate that this is not achieved via the connection of sperm to the cytophore since by this time the connections with the sperm head have been broken. However, unpublished phase contrast or dark field microscopic observations of live sperm samples at this time suggest that this may be achieved by the tendency of the distal ends of the sperm flagellum to stick together. However, exactly how this is achieved is not clear.

Ultimately the spermatozoa become totally independent, and such spermatozoa are visible in live sperm samples or samples fixed after 50 minutes incubation with SMF. The presence of free spermatozoa at 50 minutes is the basis of the in vitro bioassay, indicating the sperm morulae breakdown has taken place (Bentley, 1985).

Over the incubation period the ultrastructure of mature spermatozoa does not change, with the ultrastructure of free spermatozoa being identical to that of those which are morula-bound. Similarly, following the incubation of coelomic sperm samples with TFSW there are no free spermatozoa present after 50 minutes and observation by both light microscopy and by transmission electron microscopy show that all sperm morulae have undergone no ultrastructural changes. In addition, during experimental incubations there may be a small number of morulae which also remain undissociated after 50 minutes incubation with SMF and do not liberate free spermatozoa. Similarly, spermatocyte clusters, plates of spermatids and other developmental stages of sperm morula may also be present and these are also noted to undergo no ultrastructural changes.

\section{Other ultrastructural observations}

During the TEM observations of the fixed sperm suspension from in vitro incubations described above, cell types other than spermatozoa were visible. Coelomocytes were observed, usually in small numbers, in many of the experimental and control incubations. During incubations fixed after 40 minutes, however, they were observed often associated with free spermatozoa. Figure 15 shows an aggregate of several coelomocytes within

Fig. 12, Section of a sperm morula after $20 \mathrm{~min}$ incubation with SMF in vitro. The cytophore appears swollen and contains whorls of cytoplasm (arrowed), also the spatial distance between sperm heads has increased noticeably. Scale bar $=2 \mu \mathrm{m}$

Fig. 13. Section through a sperm morula following 30 min incubation with SMF. Relatively few sperm heads are associated with the cytophore, and the cytophore appears to be undergoing lysis. Scale bar $=2 \mu \mathrm{m}$

Fig. 14. Remains of the cytophore are visible after 40 min incubation with SMF in vitro. Scale bar = $2 \mu \mathrm{m}$ 


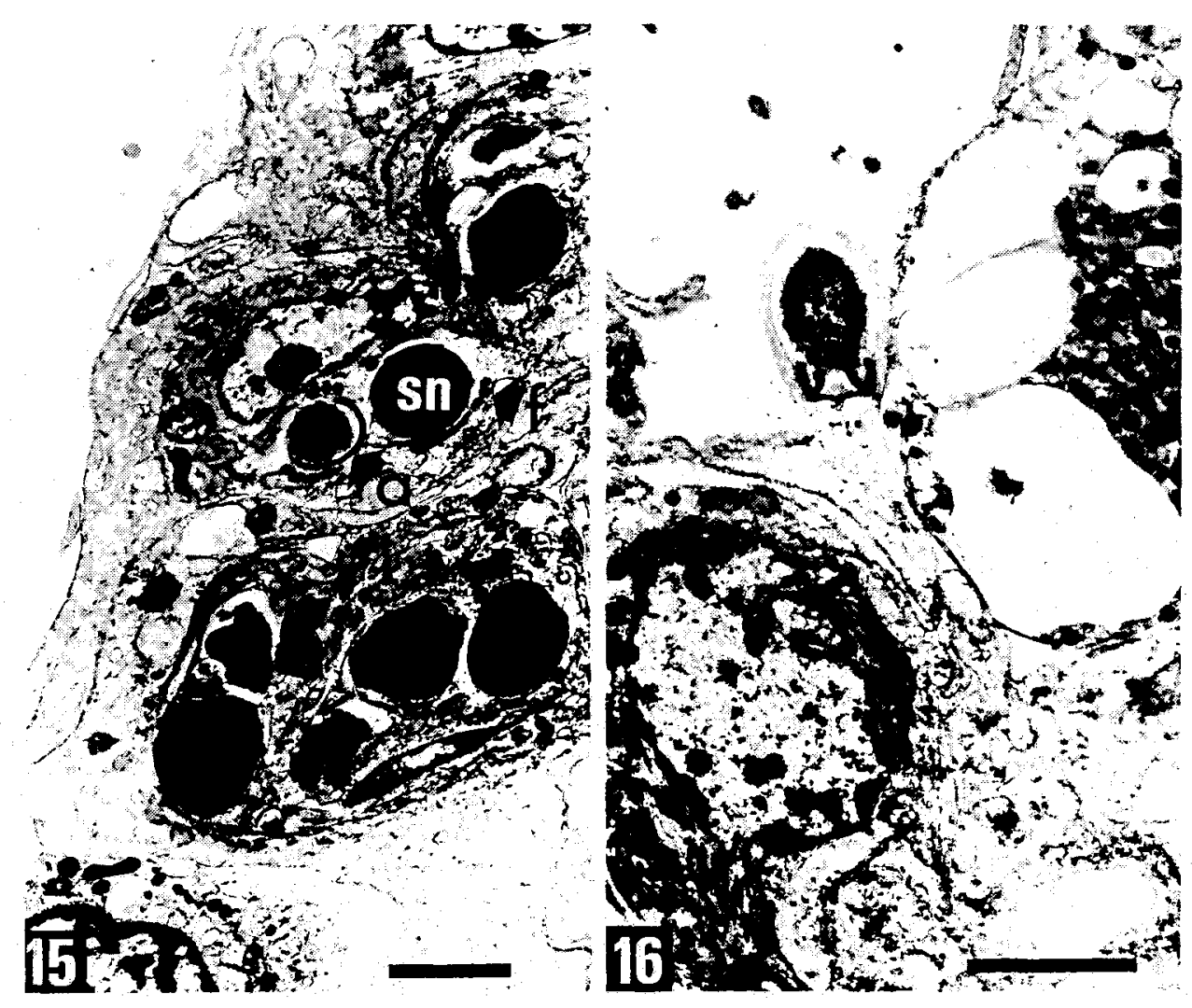

Fig. 15. Section through an aggregation of coelomocytes which contain several spermatozoa, sperm nuclei, acrosomes and flagella $(\mathrm{sn}=$ sperm nucleus; $\mathrm{a}=$ acrosome; $\mathrm{f}=$ flagella). Scale bar $=2 \mu \mathrm{m}$ Fig. 16. Section through a coelomocyte showing phagocytosed free spermatozoa. Scale bar $=10 \mu \mathrm{m}$

which the nucleus or acrosomes of seven mature spermatozoa are visible. It is not clear whether these spermatozoa have been phagocytosed by a single, or several cells, or have been encapsulated by them and are currently lying within the intercellular space. Figure 16 , however, clearly shows a spermatozoon which has been phaqocytosed by a coelomocyte.

\section{DISCUSSION}

It has been shown during previous studies on the sperm ultrastructure of Arenicola marina, using both light (Bentley, 1986a), scanning (Meijer, 1979, Bentley \& Pacey, 1989) and transmission electron microscopy (Bentley, 1986b), that the sperm type is primitive. This study, however, is more detailed and reveals much more of the internal structure of individual spermatozoa, sperm morulae, and stages of spermatogenesis. Therefore, a more thorough evaluation of the sperm biology of this species can now be made in addition to a comparison between the sperm structure of $A$. marina and the closely related Arenicola brasiliensis (Sawada, 1975). 
Sperm ultrastructure can often be related to aspects of the reproductive mode or fertilisation biology of a given species and the sperm ultrastructure of $A$. marina, for example, is typical of species which utilize external fertilisation following broadcast spawning: an oval shaped head, a simple acrosome, a single flagellum and four to six unmodified mitochondria (see Franzén, 1956). In many respects the sperm structure of $A$. marina is very similar to the sperm morphology of the closely related $A$. brasiliensis. Two morphological features of the spermatozoa of both species are worthy of note. First, both have similar acrosomal structures which are described as being like an "inverted cup" with a vesicle like structure on the apex (see Sawada [1984] for a review of the acrosome types in Polychaeta). Secondly, it has already been pointed out (Bentley \& Pacey, 1989), and this study confirms, that the sperm head of $A$. brasiliensis is more elongate than that of A. marina. The implications which can be inferred from these two observations appear to be contrary to each other. In the first instance, a similarity in the acrosome structure would suggest that the two species share similar fertilisation mechanisms (see Franzén \& Rice, 1988), whereas a difference in the shape of the sperm head, as well as leading to a more tightly packed arrangement of sperm heads around the cytophore, suggests that there may be species-specific differences in fertilisation biology; it has been suggested that where nuclear elongation occurs it may be correlated with an increase in egg size and lecithotrophic development (Franzén, 1983). This study cannot suggest any explanations for such differences, and insufficient details of egg morphology and larval development are known about either species to draw any firm conclusions.

Similar patterns of spermatogenesis between $A$. marina and $A$. brasiliensis (Sawada, 1975) have been demonstrated (see Bentley \& Pacey, 1989) and are confirmed by this study. One common feature is the formation of a sperm morula and the presence of a cytophore. The formation of a cytophore is thought to be characteristic of the annelids, but its role is not clear (Sawada, 1984). In the earthworms, Eisenia foetida and Bimastus pana, Sareen \& Verma (1983) describe the cytophore containing phospholipids, RNA, carbohydrates and proteins, but they ascribe no functional role to these substances. Sawada (1984) suggested that the cytophore may serve as a "sink" for residual cytoplasm during the division of spermatogonia, and may also serve to synchronise their development. This study shows that by the presence of endoplasmic reticulum and other organelles within the cytophore of developing spermatogonia, but not in the mature morula, the cytophore may have a nutritive or metabolic role in spermatogonial development. Cytoplasmic continuity between developing germ cells is certainly not a feature exclusive to the Annelida; intra-cellular bridges between developing spermatogonia have been observed in a number of animals, for example in the millipedes (Reger \& Cooper, 1968) and also in many mammals (Holstein \& Roosen-Runge, 1981). It has been suggested that they may facilitate physiological communication between cells (Weber \& Russell 1987), and such communication could ensure synchronous germ cell development, possibly by the spread of regulatory substances throughout the clone (Huckins, 1978). It is conceivable that the cytophore of $A$. marina could have a comparable role. The cytophore is clearly involved in the release of free spermatozoa since ultrastructural changes are closely allied with the breakdown of sperm morulae. The significance of the cytophore swelling, the role of the granular material, and the whorls of cytoplasm is not clear; although the possibility that enzymes, which are involved in the breaking of the cytoplasmic connections between spermatozoa, and substances which stimulate flagellar 
beating are being synthesised within the cytophore should not be overlooked. In addition, since sperm morula breakdown is mediated by SMF, the reception and translation of endocrine signals may well be modulated by the cytophore.

The observation that the axoneme is coiling within the flagellar membrane at its distal end is particularly interesting. Such a structure is very much like the transverse sections of paddle or discocilium which have been described in association with the sensory epithelia of a number of marine invertebrates (see Laverack, 1988). There are several variations ("club footed", "spatulate" or "paddle" type), but in general these cilia bear a discoid, biconcave or round swelling at their tip. Possible functions for such structures have been suggested, and include that they increase the membrane surface area for chemoreception (Davis \& Matera, 1982) or increase the efficiency of the power stroke (Arnolds \& Williams-Arnold, 1980). However, recent work has demonstrated that such structures are almost certainly artifacts and that they are not normally found in living organisms but are induced under a variety of ionic or hypotonic conditions (see Short \& Tamm, 1991).

Similar structures have not been widely reported as occurring in the flagellum of spermatozoa, although they have also been observed in the sperm of mammals (Drevius, 1975), some fish species (Cosson, pers. comm.) and in a number of other polychaetes species such as Nephtys hombergi and Nephtys caeca (Bentley, unpubl.), and in Harmothoe impar (Bentley \& Serries, 1992) In the case of mammalian sperm, the coiling of the flagellar axoneme occurs clearly as a result of their exposure to hypotonic media. In this study, a similar mechanism is not responsible since the fixation was isotonic to seawater. Such structures have, however, been observed in living spermatozoa of $A$. marina, under ambient osmotic conditions, using phase contrast and dark field microscopy (unpublished observations). It has been suggésted that where paddle cilia have been observed in living specimens they may occur as a result of the non-physiological conditions of the microscope slide used for observation (Short \& Tamm, 1991) and, clearly, the similarity between cilia and flagella could suggest that the coiling the flagellar axoneme may occur via a common mechanism. Any inference that such structures in the spermatozoa of $A$. marina are real should therefore be made with caution. In a previous paper (Bentley \& Pacey, 1989), scanning electron micrographs showing such structures in $A$. marina were published (see Fig. 11 in Bentley \& Pacey, 1989 ) and it was suggested that the appearance of two axonemes within a single membrane was due to the binding of axonemes from separate spermatozoa. It now seems unlikely that the pairing of flagellum in A. marina results in the binding of axonemes.

Finally, the observation that coelomocytes may phagocytose free spermatozoa, but not spermatozoa which are present in sperm morulae, is particularly interesting. Coelomocytes are known to play a vital role in the removal of unwanted cellular and noncellular material from the coelomic cavities of polychaetes (see Dales \& Dixon, 1981; Dhainaut \& Porchet-Hennere, 1989 for review). For example, it has been shown that they play a central role in the clearance of bacteria from the coelomic fluid in A marina (Fitzgerald \& Ratcliffe, 1989) and coelomocytes have been seen to proliferate following tissue damage and degeneration (Clark \& Clark, 1962). Resorption of gametes within the Polychaeta, whether due to reproductive failure or partial spawning, is a common occurrence (Olive et al, $1981 \mathrm{a}, \mathrm{b}$ ) and it has been suggested that coelomocytes clear the coelom of redundant gametes and disintegrating remains of gonadial tissue following 
spawning (Dales \& Dixon, 1981). In A. marina, unspawned gametes present within the coelomic cavity may persist for some time. De Wilde \& Berghuis (1979) noted that the coelomic cavities of $8 \%$ of a population on the Dutch coast still contained gametes (present as sperm morulae, oocytes and earlier developmental stages) over one month after spawning. In the bivalve Pecten maximus, observations of phagocytosed spermatozoa by macrophages have been made (Dorange \& Lepennec, 1989) and it has been suggested in A. marina that coelomocytes may "clean" the coelomic cavity of unspawned spermatozoa (Bentley \& Pacey, 1989). The ultrastructural evidence presented in this study suggests that there is a true role for coelomocytes in the removal of unwanted germ cells and it seems an interesting observation that only free sperm, liberated from sperm morulae, are subject to encapsulation or phagocytosis by coelomocytes. This raises interesting immunological questions with regard to how coelomocytes differentiate between free and morula bound spermatozoa. Such questions require experimental analysis.

Acknowledgements. The authors are grateful to SERC for a studentship to A.A.P., and to the technical assistance of $\mathrm{I}$. Davidson. We would also like to thank Professor M. S. Laverack for his help and assistance with the manuscript and to $\mathrm{T}$. Beddow for her work on Figure 11.

\section{IITERATURE CITED}

Arnold, J. M. \& Williams-Arnold, L. D., 1980. Development of the ciliature pattern on the embryo of the squid Loligo pealei: a scanning electron microscopy study. - Biol. Bull. mar. biol. Lab., Woods Hole 159, 102-116.

Ashworth, J. H., 1904. Arenicola. - L.M.B.C. Mem. typ. Br. mar. Pl' Anim. 11, 1-118.

Bentley, M. G., 1985. Sperm maturation response in Arenicola marina L.: An in vitro assay for sperm maturation factor and its partial purification. - Invertebr. Reprod. Dev. 8, 139-148.

Bentley, M. G., 1986a. Sperm maturation in Polychaeta. - Adv. Invertebr. Reprod. 4, 215-220.

Bentley, M. G., 1986b. Untrastructure of experimentally induced sperm maturation in Arenicola marina L. - Adv. Invertebr. Reprod. 4, 492 (abstract).

Bentley, M. G. \& Pacey, A. A., 1989. A scanning electron microscopical study of sperm development and activation in Arenicola marina L. (Annelida: Polychaeta). - Invertebr. Reprod. Dev. 15, 211-219.

Bentley, M. G. \& Pacey, A. A., 1991. Hormonal control of sperm activation and spawning in the lugworm, Arenicola marina L. (Annelida Polychaeta). - Gen. comp. Endocrínol 82, 236 (abstract).

Bentley, M. G. \& Serries, K., 1992. Sperm ultrastructure in two species of the polychaete genus Harmothoe (Polynoidae). - Helgoländer Meeresunters. 46, 170-184.

Bentley, M. G., Clark, S \& Pacey, A. A., 1990. The role of arachidonic acid and eicosatrienoic acids in the activation of spermatozoa in Arenicola marina L. (Annelida: Polychaeta). - Biol. Bull. mar. biol. Lab., Woods Hole 178, 1-9.

Clark, M. E. \& Clark, R. B, 1962. Growth and regeneration in Nephtys - Zool, Jb. (Abt. allg. Zool. Physiol. Tiere) $70,24-90$.

Dales, R. P. \& Dixon, L. R, J., 1981. Polychaetes. In. Invertebrate blood cells. Ed, by N, A Ratcliffe \& A. F. Rawley Acad. Press, New York, $1,35-74$.

Davis, W. J. \& Matera, E. M, 1982. Chemoreception in gastropod molluscs: Electron microscopy of putative receptor cells. - J. Neurobiol. $13,79-84$.

Dhainaut, A. \& Porchet-Henneré, E., 1989. Haemocytes and coelomocytes. - Microfauna mar. 4, $215-230$.

Dorange, G. \& Lepennec, M, 1989. Ultrastructural characteristics of spermatogenesis in Pecten maximus (Mollusca, Bivalvia) - Invertebr. Reprod. Dev, 15, 109-117. 
Drevius, L., 1975. Permeability of the bull-sperm membrane. In: The functional anatomy of the spermatozoon. Ed. by B. A. Afzelius. Pergamon Press, Oxford, 373-383.

Duncan, A., 1960, The spawning of Arenicola marina (L.) in the British Isles. - Proc. zool. Soc. Lond. $134,137-156$.

Eckelbarger, K. J., 1975. A light and electron microscope investigation of gametogenesis in Nicolea zostericola (Polychaeta: Terebellidae). - Mar. Biol. 30, 353-370.

Fitzgerald, S. W. \& Ratcliffe, N. A., 1989. In vivo cellular reactions and clearance of bacteria from the coelomic fluid of the marine annelid, Arenicola marina L. (Polychaeta). - J. exp. Zool. 249, 293-307.

Franzén, A., 1956. On spermiogenesis, morphology of the spermatozoon, and biology of fertilisation among invertebrates. - Zool. Bidr. Upps. 31, 355-482.

Franzén, $\AA$. 1983. Ultrastructural studies of spermatozoa in three bivalve species with notes on evolution of elongated sperm nucleus in primitive spermatozoa. - Gamete Res. 7, 199-214.

Franzén, $\AA$. \& Rice, S. A., 1988. Spermatogenesis, male gametes and gamete interactions. Microfauna mar. 4, 309-333.

Holstein, A. F. \& Roosen-Runge, E. C., 1981. Atlas of human spermatogenesis. Grosse, Berlin, $224 \mathrm{pp}$.

Howie, D. I. D., 1959. The spawning of Arenicola marina (L.). I. The breeding season. - J. mar. biol. Ass. U.K. 38, 395-406.

Howie, D. I. D., 1961. Spawning mechanism in the male lugworm. - Nature, Lond. 192, 1100-1101.

Howie, D. I. D., 1963. Experimental evidence for the humoral stimulation and ripening of the gametes and spawning in the polychaete Arenicola marina (L.). - Gen. comp. Endocrinol. 3, $660-668$.

Howie, D. I. D., 1984. The reproductive biology of the lugworm, Arenicola marina L. - Fortschr. Zool. 29, 247-263.

Huckins, C., 1978. The morphology and kinetics of spermatogonial degeneration in normal adult rats: An analysis using a simplified classification of the germinal epithelium. - Anat. Rec. 190, 905-926.

Laverack, M. S., 1988. The diversity of chemoreceptors. In: Sensory biology of aquatic animals. Ed. by J. Atema, R. R. Fay, A. N. Popper \& W. N. Tavolga. Springer, New York, 287-307.

Lewis, P. R. \& Knight, D. P., 1977. Practical methods in electron microscopy. Ed. by A. M. Glauert. Elsevier, Amsterdam, 5, 1-311.

Meijer, L., 1979. Hormonal control of oocyte maturation in Arenicola marina L. (Annelida, Polychaeta). II. Maturation and fertilization. - Dev. Growth Differ. 21, 315-329.

Newell, G. E., 1948. A contribution to our knowledge of the life history of Arenicola marina (L.). J. mar. biol. Ass. U.K. 27, 554-580.

Ochi, O., Kubo, M. \& Sawada, N., 1977. Electron microscope study on sperm differentiation in Travisia japonica (Polychaeta). - Annotnes zool. Jap. 50, 87-98.

Olive, P. J. W., 1972a. Regulation and kinetics of spermatogonial proliferation in Arenicola marina (Annelida, Polychaeta). I. The annual cycle of mitotic index in the testes. - Cell Tissue Kinet. 5 , 245-253.

Olive, P. J. W., 1972b. Regulation and kinetics of spermatogonial proliferation in Arenicola marina (Annelida, Polychaeta). II. Kinetics. - Cell Tissue Kinet, 5, 255-267.

Olive, P. J. W., 1983. Annelida - Polychaeta. In: Reproductive biology of invertebrates. Ed. by K. G. \& R. G. Adiyodi. Wiley, New York, 2, 321-341.

Olive, P. J. W., Garwood, P. R. \& Bentley, M. G., 1981a. Reproductive failure and oosorption in Polychaeta in relation to their reproductive strategies. - Bull. Soc. zool. Fr. 106, 189-196.

Olive, P. J. W., Garwood, P. R., Bentley, M. G. \& Wright, N. H., 1981b. Reproductive success, relative abundance and population structure of two species of Nephtys in an estuarine beach. - Mar. Biol. $63,189-196$.

Reger. J. F. \& Cooper, D. P., 1968. Studies on the fine structure of spermatids and spermatozoa from the millipede Polydesma sp, - J. Ultrastruct. Res, 23, 60-70.

Reynolds, E, S., 1963. The use of lead citrate at high pH as an electron-opaque stain in electron microscopy - J Cell Biol. 17, 208-212.

Sareen, M. L \& Verma, V., 1983. Morphological and cytochemical studies on the spermatogenesis in Eisenia foetida and Bimastus parva. - Res. Bull Panjab Univ. Sci. 34, 125-130. 
Sawada, N., 1975. Electron microscope studies on sperm differentiation in marine annelid worms. II. Sperm formation in Arenicola brasiliensis. - Dev. Growth Differ. 17, 89-99.

Sawada, N., 1984. Electron microscopical studies in polychaetes. - Fortschr. Zool, 29, 99-114.

Short, G. \& Tamm, S. L., 1991. On the nature of paddle cilia and discocilia. - Biol. Bull. mar. biol. Lab., Woods Hole 180, 466-474.

Smith, R. I., 1989. Notes on gamete production in Lanice conchilega (Annelida, Polychaeta, Terebellidae). - Invertebr. Reprod. Dev. 15, 7-12.

Weber, J. E. \& Russell, L. D., 1987. A study of intercellular bridges during spermatogenesis in the rat. - Am. J. Anat. 180, 1-24.

Wilde, P. A. W. J. de \& Berghuis, E. M., 1979. Spawning and gamete production in Arenicola marina in the Netherlands, Wadden Sea. - Neth. J. Sea Res. 13, 503-511. 